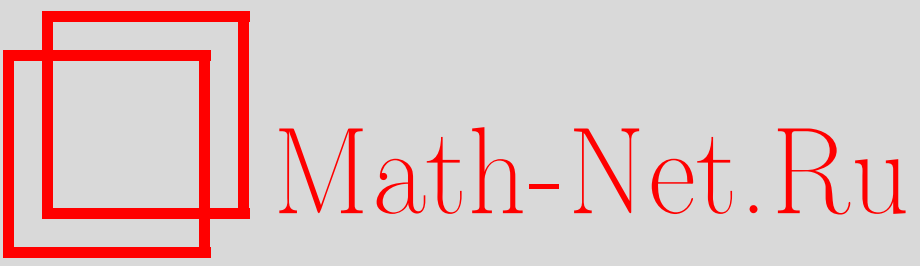

В. П. Радченко, Е. А. Андреева, О дрейфе и эффекте памяти нелинейно-упругой деформации вследствие ползучести для микронеоднородных материалов в условиях одноосного напряженного состояния, Вестн. Сам. гос. техн. ун-та. Сер. Физ.-мат. науки, 2009, выпуск 2(), 72-77

DOI: https://doi.org/10.14498/vsgtu712

Использование Общероссийского математического портала Math-Net.Ru подразумевает, что вы прочитали и согласны с пользовательским соглашением http://www . mathnet.ru/rus/agreement

Параметры загрузки:

IP : 54.196 .121 .252

26 апреля 2023 г., $17: 22: 48$ 
УДК 539.376

\title{
О ДРЕЙФЕ И ЭФФЕКТЕ ПАМЯТИ НЕЛИНЕЙНО-УПРУГОЙ ДЕФОРМАЦИИ ВСЛЕДСТВИЕ ПОЛЗУЧЕСТИ ДЛЯ МИКРОНЕОДНОРОДНЫХ МАТЕРИАЛОВ В УСЛОВИЯХ ОДНООСНОГО НАПРЯЖЕННОГО СОСТОЯНИЯ
}

\section{В. П. Радченко, Е. А. Андреева}

Самарский государственный технический университет, 443100, Самара, ул. Молодогвардейская, 244.

E-mails: radch@samgtu.ru, andreyeva@kg.ru

\begin{abstract}
Исследовано явление дрейфа нелинейно-упругой деформации вследствие ползучести в условиях одноосного напряжённого состояния на основании структурной модели типа обобщённой двухзвенной модели Максвелла. На модельном материале показано, что весъ спектр диаграмм упругого деформирования расположен между двумя стационарными состояниями: диаграммой при $t=0$ и диаграммой, соответствующей стадии установившейся ползучести, при этом мгновенно-упругая деформация проявляет одновременно свойства механической памяти и вязкоупругости. Выполнен ряд модельных расчётов, иллюстрирующих исследуемый эффект.
\end{abstract}

Ключевые слова: нелинейно-упругий материал, ползучесть, обобщённая модель Максвелла, эффект механической памяти, дрейф упругой деформачии.

1. Постановка задачи. Подавляющее большинство конструкционных и природных материалов обладают свойствами линейной упругости. На уровне механики сплошных сред общая деформация таких сред является аддитивной составляющей упругой и неупругой компонент деформации. Однако для ряда природных биокомпозитных материалов (например, костной ткани), обладающих свойством нелинейной упругости, наблюдается эффект влияния деформации ползучести на мгновенно-упругую деформацию, которая испытывает дрейф при выдержках под нагрузкой. Так, в работах $[1,2]$ при экспериментальном исследовании одноосной ползучести природного биокомпозитного материала (костной ткани) установлено, что мгновенно-упругая деформация этого материала при нагрузке $e^{\mathrm{H}}$ при $t \longrightarrow 0+0$ и полной разгрузке после ползучести при действии постоянного напряжения $e^{\mathrm{p}}$ существенно отличаются друг от друга, причём может выполняться как соотношение $e^{\mathrm{p}}>e^{\mathrm{H}}[2]$, так и $e^{\mathrm{p}}<e^{\mathrm{H}}[1]$, т. е. наблюдается эффект влияния деформации ползучести на мгновенно-упругую деформацию, при этом материал костной ткани является нелинейно-упругим.

Теоретических работ, посвященных описанию указанного эффекта, имеется незначительное число. Впервые на возможность дрейфа упругой деформации вследствие деформации ползучести было указано Ю. П. Самариным в работе [3], в которой развивался один из вариантов кинетических уравнений ползучести с позиций теории управления, однако в этой работе не детализированы условия (с точки зрения механики деформируемого твёрдого тела),

Владимир Павлович Радченко (д.ф.-м.н., проф.), зав. кафедрой, каф. прикладной математики и информатики. Елена Анатолъевна Андреева, аспирант, каф. прикладной математики и информатики. 
приводящие к отмеченному эффекту.

В работах $[4,5]$ теоретически сделаны попытки исследования в данном направлении для частного вида задач и показано, что эффект влияния ползучести на мгновенно-упругую деформацию наблюдается у слоистых композитов [4] и композитных микронеоднородных сред [5] лишь в случае нелинейного закона упругости хотя бы одной из фаз композита.

Систематический анализ этого явления для одноосного напряженного состояния на основании структурной модели микронеоднородной среды типа обобщённой двухэлементной нелинейной модели Максвелла с нелинейными упругими и вязкими элементами был выполнен в работах [6, 7]. Основной результат этих исследований сформулирован в виде двух следующих утверждений.

УтвеРЖдениЕ 1. Если локалъные элементы двухэлементной обобщённой модели типа Максвелла удовлетворяют линейному закону упругости, то упругая деформачия на макроуровне не зависит от деформачии ползучести.

УтвеРЖдениЕ 2. Если хотя бы один локалъный элемент двухэлементной обобщённой модели следует нелинейному закону упругости, то упругая макродеформация зависит от накопленной деформации ползучести.

Более детализированное исследование рассматриваемого эффекта для одноосного напряженного состояния на основе двухэлементной модели типа Максвелла, элементы которой наделены свойствами нелинейной (в общем случае) упругости и нелинейной вязкости вида

$$
\begin{gathered}
\varepsilon_{i}=e_{i}(t)+p_{i}(t), \\
e_{1}=\frac{\sigma_{1}}{E_{1}}, \quad e_{2}=\frac{\sigma_{2}\left|\sigma_{2}\right|^{n-1}}{E_{2}}, \quad \dot{p}_{i}=a_{i} \sigma_{i}\left|\sigma_{i}\right|^{m_{i}-1} \quad(i \equiv 1,2), \\
\varepsilon_{1}(t)=\varepsilon_{2}(t)=\langle\varepsilon(t)\rangle,
\end{gathered}
$$

где $\varepsilon_{i}, e_{i}, p_{i}$-соответственно полные, упругие и реологические деформации (микродеформации) в каждом из двух звеньев обобщённой модели Максвелла; $\langle\varepsilon\rangle$ - макродеформация; $n, E_{i}, m_{i}, a_{i}$ - параметры структурной модели; $\sigma_{i}$ - напряжения в элементах модели (микронапряжения), связанные с макронапряжением $\langle\sigma\rangle$ соотношением

$$
\alpha \sigma_{1}(t)+(1-\alpha) \sigma_{2}(t)=\sigma(t)
$$

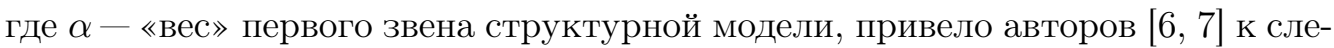
дующему утверждению.

УтвеРЖДЕнИЕ 3. Если $p_{1}^{*}<p_{2}^{*}$, где $p_{i}^{*}=p_{i}\left(t^{*}\right)(i \equiv 1,2)$ - величины деформации ползучести в локальных элементах модели, накопленные к моменту разгрузки образиа при $t=t^{*}$, то при $n>1$ для мгновенно-упругой деформации образца при нагрузке $e^{\text {н }}$ и разгрузке $e^{p}$ после ползучести при действии постоянного напря⿻сеия выполняется неравенство $e^{\text {н }}>e^{p}$, при $0<n<1-$ неравенство $e^{H}<e^{p}$, а при $n=1-e^{H}=e^{p}$.

Естественным шагом в анализе эффекта влияния ползучести на упругую деформацию является исследование характера кривой упругого деформирования в координатах $\langle e\rangle-\langle\sigma\rangle$, где $\langle e\rangle$ - упругая макродеформация, в зависимости от параметров модели (1)-(4) и длительности выдержек образца под нагрузкой. 
2. Результаты численного расчёта и анализ результатов. Численный анализ модели (1)-(4) выполнялся для режима «нагрузка - разгрузка» $(\langle\sigma\rangle=$ $=\sigma_{0}=$ const, $t \in\left[0, t^{*}\right] ;\langle\sigma\rangle=0, t>t_{*} ; t_{*}$ - время разгрузки). Расчёт одноосной ползучести по (1)-(4) осуществлялся известным методом: «шагами по времени», при этом выполнялась дискретизация области времени точками $0=t_{0}<t_{1}<t_{2}<\ldots<t_{n}<\ldots$, и на каждом интервале $\left[t_{i}, t_{i+1}\right)$ напряженное состояние в процессе ползучести полагалось постоянным, а в конце интервала при $t=t_{i+1}$ происходит упругая догрузка напряжений $\sigma_{i}=\sigma_{i}(t)$ в элементах модели в соответствии с уравнениями равновесия (4) и совместности деформаций (3).

Результаты модельных расчётов позволили выявить 3 типичных сценария кинетики диаграмм упругого деформирования вследствие ползучести, которые представлены на рис. 1-3. Значения параметров модели (1)-(4), соответствующие этим трём вариантам, приведены в таблице. Проанализируем подробно каждый из вариантов.

Параметры модели для варианта № 1 соответствуют случаю нелинейной ползучести костной ткани [7]. Здесь осуществляется расчёт при напряжении $\langle\sigma\rangle=90 \mathrm{MПа}(t \in[0,400]$ мин) и последующей разгрузке образца в течение $t \in[400,1000]$ мин. На рис. 4 приведена расчётная кривая ползучести $\langle\varepsilon\rangle=$ $=\langle\varepsilon(t)\rangle$ при данном режиме нагружения, а на рис. 1 показаны диаграммы мгновенно-упругого деформирования образца в процессе ползучести в различные моменты времени.

\begin{tabular}{c|c|c|c}
\hline \multirow{2}{*}{$\begin{array}{c}\text { Параметры } \\
\text { модели }\end{array}$} & \multicolumn{3}{|c}{ № варианта } \\
\cline { 2 - 4 } & 1 & 2 & 3 \\
\hline$E_{1}$, МПа & $3,72 \cdot 10^{4}$ & 9350 & 9500 \\
\hline$E_{2},(\mathrm{MПа})^{n}$ & $2,628 \cdot 10^{5}$ & 6000 & 6000 \\
\hline$n$ & 1,82 & 0,5 & 0,5 \\
\hline$m_{1}$ & 1 & 2 & 1 \\
\hline$m_{2}$ & 8,82 & 1 & 3 \\
\hline$a_{1},(\text { мин })^{-1}(\mathrm{MПа})^{-m_{1}}$ & $7,87 \cdot 10^{-7}$ & $4,96 \cdot 10^{-6}$ & $1,24 \cdot 10^{-8}$ \\
\hline$a_{2},(\text { мин })^{-1}(\mathrm{MПа})^{-m_{2}}$ & $7,475 \cdot 10^{-23}$ & $1,24 \cdot 10^{-8}$ & $4,96 \cdot 10^{-6}$ \\
\hline$\alpha$ & $2 / 9$ & $2 / 9$ & $2 / 9$ \\
\hline
\end{tabular}

В начальный момент приложения нагрузки $\langle\sigma\rangle=90$ МПа, когда деформация ползучести в элементах модели $p_{1}=p_{2}=0$, имеем исходную диаграмму мгновенно-упругого деформирования (цифра 1 на рис. 1), и нагружение идёт по пути $O A$. Далее в течение $t=400$ мин идёт накопление деформации ползучести $p_{1}$ и $p_{2}$ в элементах модели и одновременно происходит изменение (увеличение) значения упругой деформации при постоянном напряжении $\langle\sigma\rangle=90$ МПа (участок $A B$ на рис. 4). При разгрузке образца в момент $t=400$ мин диаграмма упругого деформирования будет уже другой (участок $B O$ на рис. 1$)$. В процессе разгрузки $(\langle\sigma\rangle=0$ при $t \in[400 ; 1000])$ происходит релаксация напряжений в элементах модели и изменение величин $p_{1}$ и $p_{2}$. И если теперь произвести нагрузку образца, например, в момент $t=500$ мин, то диаграмма упругого деформирования будет иметь вид кривой $O C$ (рис. 1 ), а при $t=600$ мин - вид кривой $O D$. По мере увеличения времени после разгрузки диаграммы упругого деформирования медленно дрейфуют от кривой 2 , соответствующей асимптотическому состоянию структурной модели (стадии установившейся ползучести), к исходной кривой 1 (см. рис. 1). 


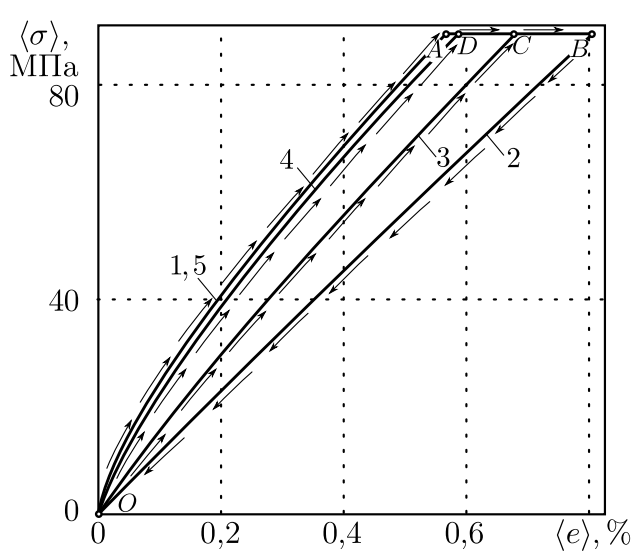

Рис. 1. Диаграммы мгновенно-упругого деформирования образца в различные моменты времени (вариант № 1): $1-t=0$; $2-t=400 ; 3-t=500 ; 4-t=600 ; 5-$ $t=800$ мин

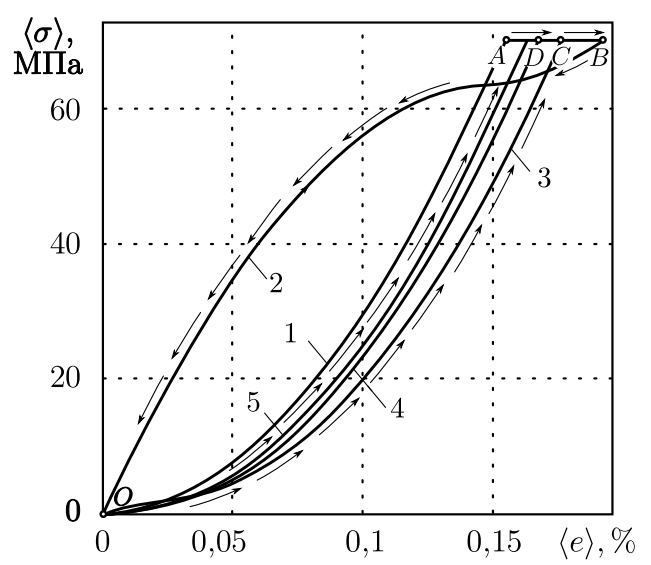

Рис. 3. Диаграммы мгновенно-упругого деформирования образца в различные моменты времени (вариант № 3): $1-t=0$; $2-t=400 ; 3-t=410 ; 4-t=500 ; 5-$ $t=1000$ мин.

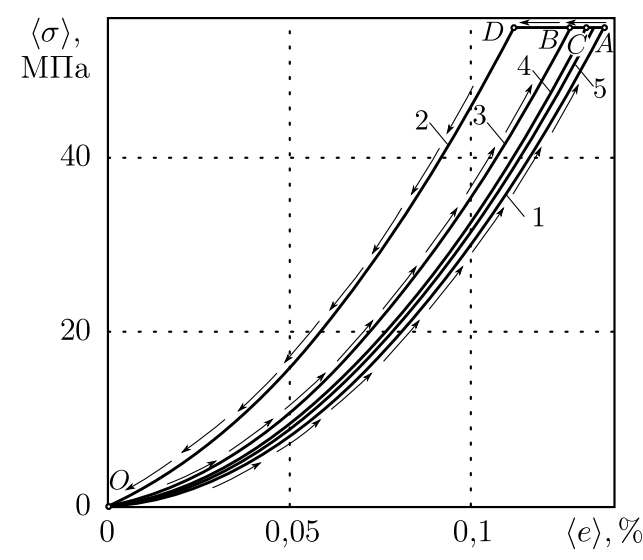

Рис. 2. Диаграммы мгновенно-упругого деформирования образца в различные моменты времени (вариант № 2): $1-t=0$; $2-t=400 ; 3-t=420 ; 4-t=500 ; 5-$ $t=800$ мин

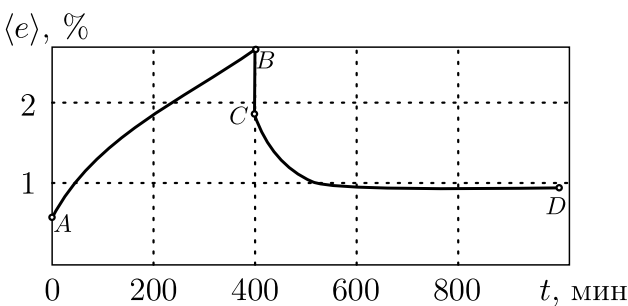

Рис. 4. Расчётная кривая ползучести для модельного материала (вариант № 1) в режиме «нагрузка - разгрузка» при $\langle\sigma\rangle=$ $=90 \mathrm{M \Pi а}$

Так, кривая 5 на рис. 1 , соответствующая времени $t=800$ мин, практически совпадает с диаграммой при $t=0$. В пределе при $t \rightarrow \infty$ остаточные напряжения в процессе разгрузки релаксируют полностью, и происходит полное восстановление первоначальных упругих свойств материала.

Аналогичные результаты получены и для вариантов № 2 и № 3, в которых производится расчёт при напряжениях $\langle\sigma\rangle=55 \mathrm{MПа} \mathrm{и}\langle\sigma\rangle=70 \mathrm{MПа} \mathrm{в}$ течение $t \in[0 ; 400]$ мин с последующей разгрузкой при $t \in[400 ; 1000]$ мин (см. рис. 2 и рис. 3 ), при этом характер «выпуклости - вогнутости» диаграмм $\langle\sigma\rangle-\langle e\rangle$ качественно отличается от соответствующих диаграмм на рис. 1. 
3. Выводы. Выполненные исследования позволяют сделать следующие выводы:

1) для нелинейно-упругих материалов происходит медленный дрейф диаграммы упругого деформирования $\langle e\rangle-\langle\sigma\rangle$ вследствие ползучести, при этом весь спектр диаграмм расположен между двумя стационарными состояниями, а именно диаграммой при $t=0$ и диаграммой, соответствующей асимптотическому состоянию структурной модели под нагрузкой при $t \rightarrow \infty$ (стадии установившейся ползучести);

2) в процессе дрейфа мгновенно-упругая деформация проявляет свойства механической памяти, поскольку в процессе разгрузки образца (при $t \rightarrow \infty)$ происходит полное восстановление первоначальных упругих свойств, а также вязкоупругости, поскольку мгновенно-упругая деформация неявно (через накопленную деформацию ползучести) зависит от времени.

Таким образом, при ползучести образцов из нелинейно-упругого материала в течении некоторого интервала времени при постоянном напряжении наблюдаются своеобразные «гистерезисные петли» на диаграмме упругого деформирования $\langle e\rangle-\langle\sigma\rangle$.

Работа выполнена при поддержке РФФИ (проект № 07-01-00478-а).

\section{БИБЛИОГРАФИЧЕСКИЙ СПИСОК}

1. Кнетс И. В., Вилкс Ю. К. Ползучесть компактной костной ткани человека при растяжении // Механика полимеров, 1975. - Т. 11, № 4. - С. 634-638.

2. Мелнис А. Э., Лайзан Я. Б. Нелинейная ползучесть компактной костной ткани человека при растяжении // Механика полимеров, 1978. - Т. 14, № 1. - С. 97-100.

3. Самарин Ю. П. О применении теории управления к исследованию ползучести конструкций / В сб.: Механика деформируемых сред. - Куйбышев: Изд-во Куйбышев. ун-та, 1976. - C. $123-129$.

4. Радченко В. П., Самарин Ю. П. Влияние ползучести на величину упругой деформации слоистого композита // Механика композитных материалов, 1983. - Т. 19, № 2. C. 231-237.

5. Радченко В. П. Об одной структурной реологической модели нелинейно-упругого материала // Прикладнал механика, 1990. - Т. 26, №6. - С. 67-74.

6. Радченко В. П., Шапиевский Д. В. О дрейфе упругой деформации для нелинейно-упругих материалов вследствие ползучести // Вестн. Сам. гос. техн. ун-та. Сер. Физ.мат. науки, 2006. - № 43. - С. 99-106.

7. Радченко В.П., Шапиевский Д. В. Математическая модель ползучести микронеоднородного нелинейно-упругого материала // ПМТФ, 2008. - Т. 49, № 3. - С. 157-163.

Поступила в редакцию 20/VII/2009; в окончательном варианте $-18 / \mathrm{X} / 2009$. 
MSC: 74E35, 74B20, 74A20

ON DRIFT AND MEMORY EFFECT OF NONLINEAR ELASTIC

STRAIN ARISING DUE TO CREEP FOR MICRONON-UNIFORM MATERIALS IN CONDITIONS OF LINEAR STRESS

\section{V.P. Radchenko, E. A. Andreeva}

Samara State Technical University,

244, Molodogvardeyskaya str., Samara, 443100.

E-mails: radch@samgtu.ru, andreyeva@kg.ru

The drift phenomenon of nonlinear elastic strain arising due to creep in conditions of linear stress on the basis of structural model of Maxvilles generalized two-tier model type is studied. On the basis of modeling material it is demonstrated that is the whole spectrum of diagrams of elastic strain is located between two stationary conditions: the diagram at $t=0$ and the diagram corresponding to the stage of established creep, thus instantly-elastic deformation shows simultaneously the properties of mechanical memory and viscoelasticity. The modeling calculations illustrating the effect investigated is executed.

Key words: nonlinear materials, creep, Maxville's generic model, mechanical memory effect, elastic strain drift.

Original article submitted 20/VII/2009; revision submitted $18 / \mathrm{X} / 2009$.

Vladimir P. Radchenko (Dr. Sci (Phis. \& Math.)), Head of Dept., Dept. of Applied Mathematics \& Computer Science. Elena A. Andreeva, Postgraduate Student, Dept. of Applied Mathematics \& Computer Science. 